\title{
Probleme alla bolognese: Juristenausbildung und Bologna
}

\author{
Thomas Pfeiffer"
}

\section{Einleitung}

Juristenausbildung und Bologna - zu dieser nahezu endlosen Diskussion scheint schon fast alles gesagt. Doch gleichwohl gibt es gleich doppelten Anlass zu einer erneuten Stellungnahme, ${ }^{1}$ nämlich erstens den Koalitionsvertrag zwischen CDU/CSU und F.D.P. und zweitens die aktuellen Studentenproteste des Herbstes 2009.

Während der „schwarz-rote“ Koalitionsvertrag für die 16. Wahlperiode des Deutschen Bundestags einer Übertragung des Bologna-Modells auf die Juristenausbildung eine Absage erteilte, ist der aktuelle Koalitionsvertrag zwischen CDU/CSU und FDP weitaus offener gehalten. Unter der Überschrift „Juristenausbildung“ heißt es dort: „Der Bologna-Prozess stellt die Juristenausbildung in Deutschland vor besondere Probleme. Der hohe Qualitätsstandard der Ausbildung, wissenschaftliche Tiefe, thematische Vielfalt und Praxisorientierung müssen auch künftig Maßstab für die Studienabschlüsse sein.“

Diese Besonderheiten der Juristenausbildung müssen zudem vor dem Hintergrund der allgemeinen Diskussion zu sehen, die die deutsche Hochschullandschaft erfasst hat und in der die mit dem Schlagwort „Bologna“ verbundene Umstrukturierung erneut reflektiert wird.

Über diese aktuellen Anlässe hinaus ist als neuere Entwicklung zu konstatieren, dass an einzelnen Universitäten - oft, aber keineswegs immer parallel zu einem Staatsexamensstudiengang - Bachelor- und Masterprogramme eingerichtet wurden, was die Bewertung des Für und Wider einer Erstreckung des Bologna-Prozesses auf die Juristenausbildung nicht unbeeinflusst lassen kann.

\section{Verschiedene Bologna-Grundmodelle}

Trotz des Anlasses zu neuer Diskussion bleibt freilich an der Ausgangslage vieles unverändert, vor allem ein Kernaspekt, zu dem sich auch der Koalitionsvertrag wohl dem Grundsatz nach bekennen will: Der Zugang zu den Justizberufen muss umfassend wissenschaftlich vorgebildeten und praktisch vorbereiteten Juristen überlassen bleiben. Das Ziel einer umfassenden wissenschaftlichen Vorbildung ist im Vergleich zum Staatsexamensstudiengang in einem dreijährigen Bachelorstudiengang nicht oder jedenfalls nur unter erheblichen Qualitätsverlusten erreichbar.

* Prof. Dr. Thomas Pfeiffer lehrt Bürgerliches Recht, Internationales Privatrecht, Rechtsvergleichung und Internationales Verfahrensrecht an der Ruprecht-Karls-Universität Heidelberg.

1 Der Verfasser hat seine Position in einer früheren Phase der Diskussion bereits einmal dargelegt und verweist darauf: T. Pfeiffer, Wird der Juristenausbildung der Bologna-Prozess gemacht?, NJW 2005, $2281 \mathrm{f}$. 
Wer einen dreijährigen Bachelorstudiengang als juristischen Regelabschluss propagiert, muss deshalb letztlich dafür eintreten oder in Kauf nehmen, dass einer Mehrheit der Absolventen der Zugang zur eigentlichen juristischen Berufsausübung versperrt bleibt.

Das ist weitgehend unbestritten. Die verschiedenen Modelle einer Bolognarisierung der Juristenausbildung ziehen hieraus allerdings unterschiedliche Konsequenzen:

\section{Zweigestufte Ausbildung mit Staatsprüfungen bei Übertragung der Bolognastruktur auf das Studium}

Eine des Öfteren erwogene oder propagierte Modellstruktur will (mit unterschiedlichen Einzelausprägungen) einerseits das Grundprinzip der zweigestuften, aus Studium und Vorbereitungsdienst bestehenden Juristenausbildung mit Staatsprüfungen beibehalten, aber zusätzlich auf den Studiumsteil der Ausbildung die Bolognastruktur übertragen. Hierauf zielt etwa die Position der Bundesrechtsanwaltskammmer. ${ }^{2}$

Besondere Beachtung erlangen in diesem Zusammenhang auch die Überlegungen der nordrhein-westfälischen Justizministerin Müller-Piepenkötter. ${ }^{3}$ Ihrem Modell liegt die Vorstellung zugrunde, dass die Mehrheit der Studierenden das Studium nach 3 Jahren mit einem Bachelorabschluss beendet und keinen eigentlich juristischen Beruf ergreift. Auf dieser Grundlage sollen alsdann ein Masterstudiengang mit Abschluss (verbunden mit einer Justizeingangsprüfung) und ein Vorbereitungsdienst mit großem (oder zweitem) Staatsexamen folgen.

\section{Modelle eines vierjährigen Bachelor}

Ein anderes Grundmodell ist das sogenannte Vierstufenmodell mit einem vierjährigen Bachelor. Das Modell eines vierjährigen Bachelor, wie es etwa Jeep in die Diskussion gebracht ${ }^{4}$ und wie es in etwas abweichender Weise auch den Vorschlägen des Deutschen Anwaltvereins ${ }^{5}$ zugrunde liegt, steht der gegenwärtigen Juristenausbildung näher. Die Bachelorprüfung steht hier am Ende eines vierjährigen Studiums, das namentlich die Kernfächer umfasst. Es bildet zugleich die Voraussetzung für die Zulassung zum Staatsexamen, das hierauf aufbaut. Bei Jeep tritt die Masterprüfung an die Stelle der bisherigen, kürzlich erst eingeführten Schwerpunktbereichsprüfun-

2 Presseerklärung der Bundesrechtsanwaltskammer vom 15.7.2007: Bundesrechtsanwaltslammer betont große Bedeutung der einheitlichen juristischen Ausbildung, http://www.brak.de/seiten/04_07_19.php, 7.12.2009.

3 Dazu das Papier des JM-NRW: Das nordrhein-westfälische Modell zur Umsetzung des Bologna-Prozesses in der deutschen Juristenausbildung, http:/www.justiz.nrw.de/JM/justizpolitik/schwerpunkte/juristenausbildung/bologna_prozess/langfassung.pdf, 7.12.2009.

4 J. Jeep, Der Bologna-Prozess als Chance - Warum die Juristenausbildung durch Bachelor und Master noch besser werden kann, NJW 2005, $2283 \mathrm{f}$.

55 Thesen des DAV zur Umsetzung des Bologna-Prozesses in der Juristenausbildung http://anwaltverein.de/downloads/pressemitteilungen/Bologna.pdf, 10.12.2008. 
gen und wird vom Bachelorabschluss sowie dem Staatsexamen abgekoppelt. An das verkürzte Referendariat schließt sich ein Zweites Staatsexamen an.

Dem DAV-Modell geht es offenbar vor allem um die Schaffung eines vierjährigen qualifizierten und qualifizierenden Bachelorabschlusses, um die Einführung des dort favorisierten Spartenmodells des Vorbereitungsdienstes (jeweils getrennte Vorbereitungsdienste für Anwaltschaft, Justiz und Verwaltung) zu ermöglichen, dem ersichtlich auch das berufsständische Interesse an einer Begrenzung des Zugangs zu den juristischen Berufen zugrunde liegt.

\section{Kriterien einer Bewertung}

\section{1. Äußerliches}

\section{a) Verlängerung oder Verkürzung der Ausbildungszeit}

Von den vielen Effekten, die solche Modelle bewirken werden, lassen sich manche recht sicher erkennen; andere lassen sich lediglich prognostizieren. Sicher vorhersehbar ist:

Nach den letzten verfügbaren Zahlen betrug etwa die durchschnittliche Studiendauer im Lande Baden-Württemberg bis zum ersten Staatsexamen 9,66 Semester (Wiederholer eingerechnet). Bei den Erstteilnehmern betrug die Dauer 9,10 Semester, bei denjenigen, die bestanden haben, sogar nur 8,73 Semester. ${ }^{6}$ Würde nun eine dreiplus zweijährige Studienstruktur zwingend vorgegeben, so wäre die jetzt den Regelfall bildende Studiendauer von 8-9 Semestern bis zur ersten Prüfung obsolet, weil sich ein Bachelor-Studium mit anschließendem Master in einem kürzeren Zeitraum als innerhalb von 5 Jahren praktisch nicht absolvieren lassen wird. Im Gegenteil: In der allgemeinen Diskussion über den Bolognaprozess bricht sich allmählich die Erkenntnis Bahn, dass die vorgesehenen 3 Jahre bis zum Bachelorabschluss eine Regelstudienzeit bilden, die Überschreitungen keineswegs ausschließt. Für alle praktischen Zwecke ist es deshalb unabweisbar, dass die durchschnittliche Studiendauer bis zur ersten (Staats-)Prüfung bis zum Staatsexamen auf der Grundlage eines 3+2Jahre-Modells wieder anstiege, was alle durchaus ertragreichen Anstrengungen um eine Verkürzung der Ausbildungszeiten (G12 und sinkende Verweildauer der Jurastudenten an den Universitäten in den letzten Jahren) konterkarierte.

Nun mag man sich mit der Studienzeitverkürzung für diejenigen trösten, die nach dem Bachelorabschluss nicht den Weg zur Ausübung von Juristenberufen weitergehen. Eine Aufrechnung von kürzeren Bachelorstudienzeiten und einem längeren Weg zu Staatsexamen sollte sich indessen verbieten. Wenn man die Ausbildungszeiten

6 Bericht des Landesjustizprüfungsamts des Landes Baden-Württemberg für das Jahr 2007, http:// www.jum.baden-wuerttemberg.de/servlet/PB/show/1219496/Jahresbericht\%202007.pdf.7.12.2009, S. 4 . 
deutscher Volljuristen als Problem sehen will, dann sind sie das insbesondere auch für angehende Anwälte.

Eine schlichte Übertragung des 3+2-Jahre-Modells auf den Studienteil der Ausbildung bei Beibehaltung der zweistufigen Gestaltung von Studium und Vorbereitungsdienst sollte sich schon aus diesem Grund verbieten.

\section{b) Hinabstufung der Referendare auf Bachelorniveau}

Das Jeep-Modell führt nicht zu derselben zeitlichen Ausdehnung wie das Modell eines dreijährigen Bachelors, sondern zu anderen Bedenken:

Die bisherige erste juristische Prüfung wird aufgelöst in ein Staatsexamen und einen Masterabschluss, wobei letzterer nicht obligatorisch ist. Hervorhebenswert ist dabei, dass - wenn man Funktionsäquivalente des bisherigen Systems einerseits und des Bolognamodells andererseits definieren will - die derzeitige erste juristische Prüfung die Anforderungen des Bachelor und des Masterabschlusses vereint. Und in der Nomenklatur des Bolognaprozesses ist deshalb die Feststellung sicher angemessen, dass die derzeitigen Staatsexamensabschlüsse sich auf dem Niveau eines Masterabschlusses bewegen. Davon geht auch das Jeep-Modell aus, indem es die bisherige erste Prüfung in einen Bachelor- und einen Masterteil spaltet. Das bedeutet freilich umgekehrt: Im Jeep-Modell verfügen nicht mehr alle Absolventen über das bisherige Master-Niveau.

\section{c) Zum DAV-Modell: Niveauabsenkung oder Etikettenschwindel zum Nachteil der Absolventen?}

Das Modell des DAV ist, soweit aus zugänglichen Quellen ersichtlich, zur Frage der Inhalte des Bachelorabschlusses weniger klar. Auch hier ergibt sich indessen aus dem Umstand, dass die bisherigen Absolventen des ersten Examens sich auf der Ebene des Master bewegen (wenn diese These zutrifft - und sie trifft zu), ein durchgreifender Einwand. Wenn man das erste Examen wie der DAV an einen Bachelorabschluss knüpfen will, gibt es nur zwei Möglichkeiten: nämlich eine Niveauabsenkung, was der DAV von sich weist, oder ein Etikettenschwindel zum Nachteil der Absolventen.

\section{d) Weitere Einwände}

Die weiteren Einwände gegen ein solches Modell sind nicht neu: Sie betreffen einmal die hier nicht zur vertiefende Frage nach den langfristigen Folgen des mit einem Spartenmodell der Juristenausbildung verbundenen Verlusts der einheitsjuristischen Ausbildung und Mobilität zwischen den Berufen. Zum anderen geht es um die Frage, ob der Markt die betreffenden Bachelor aufnehmen kann und will; sie ist auch bei freundlicher Einschätzung noch offen. Bei rationalem Kalkül werden sich die meisten Absolventen den Zugang zur volljuristischen Ausbildung nicht nehmen lassen; darauf deuten auch ausländische Erfahrungen hin. 
Völlig unklar bleibt zudem, wie die Universitäten ein solches Modell der Kapazität nach bewältigen sollen. Die derzeitigen Staatsexamensstudiengänge sind auf ein 7semestriges Studium angelegt. 3 Jahre Bachelorstudium und 2 Jahre Masterstudium ergeben 10 Semester, 4 Jahre Bachelor und $1 \mathrm{Jahr}$ Master übrigens auch. Woher die Universitäten die zusätzlichen Mittel und Kapazitäten nehmen sollen, um diese Ausweitung des Programms um drei Semester zu bewältigen, bleibt unklar. Diese Frage stellt sich nur dann nicht, wenn man die Hinabstufung der Juristen zu Bachelorn, sei es dem Niveau nach oder im Rahmen eines Etikettenschwindels zum Nachteil der Absolventen, für erstrebenswert hält.

Kein hinreichender Ausgleich für den sonst entstehenden Zusatzaufwand ergibt sich jedenfalls aus dem Umstand, dass nach dem NRW-Modell $60 \%$ der Absolventen nach dem Bachelor nicht den spezifisch juristischen Masterabschluss erwerben sollen. Dass ein solcher Ausgleich rechnerisch nicht funktionieren kann, erhellt schon daraus, dass die Zahl der Studierenden in sechs von (bisher) sieben Semestern von dieser Maßnahme unbeeinflusst bleibt.

Zudem müssen Universitäten neben den rechtlichen Kapazitätsmaßgaben auch ihre faktische Lehrkapazität im Blick haben. Deren Inanspruchnahme wiederum bliebe namentlich in den Master-Semestern von einer geringeren Zahl von Studenten pro Semester weitgehend unbeeinflusst. Das wäre nur dann anders, wenn man sich durch eine geringere Zahl von Teilnehmern ersparte, Veranstaltungen zu teilen, die gegenwärtig aufgrund ihrer Größe geteilt werden. Die Teilung von Veranstaltungen aufgrund ihrer schieren Größe ist in fortgeschrittenen Jura-Semestern oder bereits bestehenden Masterstudiengängen indessen ein praktisch inexistentes Phänomen.

Es bliebe noch, aufgrund der geringeren Größe der Masterstudiengänge auf eine geringere Prüfungsbelastung zu hoffen. Allein, auch diese Hoffnung wird sich als Illusion entpuppen. Mit dem Bolognaprozess untrennbar verbunden ist nun einmal die Modularisierung von Studiengängen und die einher gehende Schaffung studienbegleitender Prüfungen. Selbst wenn man der Versuchung widersagt, jede Veranstaltung mit einer Prüfung zu versehen, und von der Möglichkeit breitflächiger Modulprüfungen großzügig Gebrauch macht, ist ein dramatisches Ansteigen der Universitätsprüfungen im Falle der Bolognarisierung unvermeidbar. Zum Staatsexamen kommen bei einem solchen Modell noch zwei universitäre Abschlussprüfungen und außerdem noch die erforderlichen Modulprüfungen hinzu. Es ist leicht vorhersehbar, dass der Prüfungsaufwand erheblich ansteigen wird. Zeit und Kraft sind jedoch begrenzt. Das trifft selbst auf Hochschullehrer zu. Deshalb kann und muss ein solcher zusätzlicher Prüfungsaufwand auf Kosten der Leistungsfähigkeit in Forschung und Lehre gehen. 
Wer der Vorstellung anhängen sollte, eingesparte Mittel in den Justizhaushalten wegen einer geringeren Zahl der Staatsexamensabsolventen ließen sich an die juristischen Fakultäten „umleiten“ und damit ein Ausgleich schaffen, dem müsste Ahnungslosigkeit in vielfacher Hinsicht bescheinigt werden. Das betrifft sowohl die Mechanismen der Mittelverteilung zwischen den Ressorts in den jeweiligen Bundesländern als auch diejenigen der Mittelverteilung zwischen den Hochschulen eines Bundeslandes als auch die innerhalb der Universitäten heute üblichen Steuer- und Lenkungsmechanismen bei der Budgetverteilung. Und selbst wenn es gelänge (was ausgeschlossen ist), würde es angesichts der unvermeidlichen Prüfungsvermehrung für einen Ausgleich nicht ausreichen. Die Vorstellung, durch ein bolognarisiertes Modell könne die Wissenschaftlichkeit des juristischen Studiums gestärkt werden, geht an den Rahmenbedingungen, unter denen Universitäten heute arbeiten, in vielfacher Hinsicht vorbei. Es kann aus Sicht des Rektoratsmitglieds einer Universität nur als (leider oder gottseidank) eher wirklichkeitsfern bezeichnet werden.

Wäre es gleichwohl - hypothetisch - tatsächlich erreichbar, dass zusätzliche Mittel in die universitäre Juristenausbildung fließen, dann bedürfte es einer intensiven Diskussion, an welcher Stelle diese am besten investiert werden. Man mag vertreten, dass in der juristischen Ausbildung manches fehlt. Was sicher nicht fehlt, ist, sind intensive Prüfungen. Die beiden Staatsexamina durch eine Bachelor und eine Masterprüfung zu ergänzen zu wollen, führt in die Irre, aber nicht zu mehr Qualität.

Kurz gesagt: Ein wesentlicher Geburtsfehler solcher Modelle besteht darin, dass man beides zugleich - Beibehaltung der Qualitätssicherungsfunktion des Staatsexamens bei gleichzeitiger Einführung zusätzlicher Abschlüsse, die nicht nur auf dem Papier stehen - kostenlos nicht haben kann.

\section{Inhaltliches}

\section{a) Strukturierung, Modularisierung, Kompetenzorientierung?}

Als wesentlicher Vorzug des Bolognaprozesses wird in der Debatte um Bologna angeführt, dass er - namentlich in den Geisteswissenschaften - zu einer klareren Strukturierung und geringeren Abbrecherquoten führe. ${ }^{7}$ Diese Einschätzung beruht ersichtlich darauf, dass in manchen geisteswissenschaftlichen Fächern ein fixierter Kanon der zum Abschluss benötigten Lehrinhalte fehlt. Die Bolognamethode der Modularisierung ist auch und vor allem der Versuch einer Reaktion hierauf. Freilich beruht sie auf der Annahme, die Inhalte eines Studiums ließen sich in einzeln abfragbare „Kompetenzen“ zerlegen.

Das geht an den Erfordernissen der Rechtswissenschaft indessen gleich mehrfach glatt vorbei: Erstens ist der zum ersten Examen führende Kanon und die dorthin

7 Etwa A. Schavan, in einem Interview mit Zeit-online/Tagesspiegel vom 6.12.2009, http://www.zeit.de/ politik/deutschland/2009-12/schavan-interview, 15.12.2009. 
führende Struktur in den Kernfächern ebenso klar wie allgemein bekannt. Zweitens führt die Zerlegung der Rechtswissenschaft in Einzelkompetenzen auch fachlich in die falsche Richtung. Denn zwar kann man die Methode rechtswissenschaftlichen Arbeitens anhand einzelner fachlich begrenzter Übungen kennen lernen. Den für den umfassend gebildeten und ausgebildeten Juristen notwendigen Blick auf die Rechtsordnung als Ganze lässt sich bei Aufrechterhaltung der gegenwärtigen Qualitätsmaßstäbe nur durch Aufgabenstellungen auf der Niveaustufe des derzeit bestehenden Examens prüfen.

Die durch den Bolognaprozess verlangte Modularisierung führt vor diesem Hintergrund unweigerlich vor die Alternative, den studienbegleitenden Prüfungsaufwand der modulbezogenen Kompetenzüberprüfung mit den Anstrengungen des ersten juristischen Examens zu kombinieren, oder dazu, dieses Examen abzuwerten. Ersteres bewirkte, wie deshalb erneut zu bemerken ist, eine Aufblähung der Prüfungslast, zweites eine Verschiebung der Gewichte hin zu weniger anspruchsvollen Prüfungen.

\section{b) Die Vorzüge des Staatsexamens}

Als Tertium bliebe nur die Aufgabe des Staatsexamens als Voraussetzung für den Vorbereitungsdienst. Diese will aber keines der im Blickpunkt stehenden Modelle, und zwar aus guten Gründen.

Die deutsche Staatsexamenskultur ist ein im internationalen Vergleich hohes Gut von kaum ersetzlichem Wert über das juristische Studium hinaus. Das Staatsexamen steht für bundesweit weithin vergleichbare und verlässliche Abschlüsse. Das Zusammenwirken von Wissenschaftlern und Praktikern im Staatsexamen bildet die Grundlage für den in Deutschland ebenso engen wie fruchtbringenden Dialog zwischen Gerichten und Wissenschaft. Vor allem jedoch bildet dieses Examenssystem die Studierenden früh- und rechtzeitig auf eine Reproduktion vorhersehbarer Ergebnisse hin aus; es bildet deshalb eine unverzichtbare Grundlage dafür, dass Gerichtsentscheidungen in Deutschland - jedenfalls nach den Erfahrungen des Autors aus der Gutachtenspraxis des Heidelberger Instituts für ausländisches und internationales Privat- und Wirtschaftsrechts - besser vorhersehbar sind als in vielen anderen Rechtsordnungen. Selbstverständlich sind rechtsstaatliche Bedingungen auch ohne Staatsexamenssystem aufrecht zu erhalten. Allein, das Bessere ist der Feind des Guten, und die bessere Vorbereitung auf vorhersehbare Ergebnisse verbürgt das Staatsexamenssystem.

Es bleibt ein Alleinstellungsmerkmal der Angehörigen juristischer Berufe, dass eine von drei Staatsgewalten, die Judikative, ausschließlich ihnen anvertraut ist. Die zur Ausübung dieser Berufe berechtigende und befähigende Ausbildung steht deshalb in einer mit allen anderen Berufen (auch mit solchen, die bisher ebenfalls ein Staatsexamenssystem kennen) nicht zu vergleichenden Weise in einer öffentlichen Verantwortung. Das derzeitige Staatsexamenssystem spiegelt just diese Sondersituation in 
einer durch andere Ausbildungsmodelle nur eingeschränkt substituierbaren Weise wider. Ein in diesem Punkt vergleichbarer Ersatz ist schlicht nicht ersichtlich.

\section{c) Internationalisierung}

Dass die internationale Mobilität der Bachelorstudierenden gering ist, kann inzwischen auch über Deutschland hinaus als Gemeinplatz gelten. Das ist auch wenig überraschend, weil den anglo-amerikanischen Vorbildern dieses Modells die kontinentaleuropäische Tradition der fahrenden Scholaren fremd ist und vielmehr eine ausgeprägte Kultur der Verbundenheit mit der eigenen Hochschule gepflegt wird. Der internationale Wechsel erfolgt in der Bologna-Welt vor allem beim Übergang vom Bachelor zum Master. Unter den Bedingungen von nach wie vor dominierenden nationalen Besonderheiten ist der Masterabschluss aber jedenfalls dann nur schwer vollständig ins Ausland zu verlagern, wenn man am Ziel der Masterqualifikation für Volljuristen festhalten will. Nun ließen sich die Bolognastrukturen auch so gestalten, dass sie eine größere Mobilität eröffnen. Für die typischerweise von Juristen nachgefragten Formen des Auslandsaufenthalts ist Bologna indessen kein Gewinn.

\section{d) Vom Sinn von Abstimmungen mit den Füßen}

Wie bereits angedeutet, ist die Entwicklung der Juristenausbildung in Deutschland auch davon geprägt, dass sich im Rahmen eines pluralistischen Ausbildungsmodells $^{8}$ an einer Reihe von deutschen Fakultäten Bachelor- und Masterstudiengänge mit unterschiedlicher Ausrichtung und Struktur gebildet haben. Da sich die Richtigkeit und Überlegenheit unterschiedlicher Modelle nicht im naturwissenschaftlichen Sinne beweisen lässt, sind andere Mechanismen der Vergewisserung über deren Qualität erforderlich.

„Exit“ und „Voice“, jene zentralen Einflussmöglichkeiten des Einzelnen im politischen Diskurs, ${ }^{9}$ deuten auf zweierlei hin. Erstens haben die aktuelle Diskussion und die aktuelle Kritik am Bolognaprozess („Voice“) Gründe und Ursachen, deren Kern in der Frage liegt, inwieweit die die Einheitlichkeit und spezifische Struktur des Bolognamodells die richtige Antwort auf die sich in verschiedenen Studiengängen stellenden unterschiedlichen Fragen darstellt. Es wäre nicht ohne Ironie, wenn die Juristenausbildung ausgerechnet zu einem Zeitpunkt dem Bolognaprozess unterworfen würde, zu dem allenthalben darüber diskutiert wird, wie seine bisherigen Auswirkungen am besten begrenzt oder beseitigt werden können.

Zweitens erlaubt das bestehende und sich erweiternde Modell einer pluralistischen Juristenausbildung den Wettbewerb um das beste Modell und den Studierenden eine Abstimmung mit den Füßen. Wer die Ausbildung für die spezifischen Juristenberufe

8 Dazu Beschluss des 87. Deutschen Juristenfakultätentages, Juristenausbildung und Bologna-Prozess (DJFT 2007/I), http://www.djft.de, 16.12.2009 unter „Beschlüsse“, „Juristenausbildung“).

9 Grundlegend A. O. Hirschmann, Exit, Voice and Loyalty, Cambridge/MA: Harvard University Press 1970 . 
bundesweit einheitlich den Maßgaben des Bolognamodells unterwerfen möchte, kann nicht umhin, eine solche Abstimmung auszuschließen. Das wäre nicht nur unklug, weil es den besten Richtigkeitsindikator abschneidet, über den wir insoweit verfügen. Es würde vor allem die Studierenden, aber in gewissem Maße auch die Hochschullehrer, ihrer wichtigsten Einfluss- und Selbstbestimmungsmöglichkeit („Exit“) berauben, indem die sonst mögliche Abstimmung mit den Füßen vereitelt wird.

\section{Statt eines Schlusses}

Es ist eine Grunderkenntnis komparatistischen Arbeitens, dass die Rezeption von Modellen und Regeln aus anderen Kontexten niemals zu einer exakten Übernahme, sondern regelmäßig zu einer Rekonstruktion unter den spezifischen Bedingungen des rezipierenden Systems führt. Stets stellt sich deshalb die Frage, welche neuen und im Herkunftssystem unbekannten Pathologien solch eine Rezeption verursacht. Die vorstehenden Überlegungen haben einige dieser Pathologien, soweit sie offensichtlich sind, aufgezeigt.

Da nicht zu erwarten ist, dass sich die politische Diskussion dem ganz verschließen wird, gehört nicht sehr viel an Phantasie zu der Erwartung, dass in Bälde verschiedene Vorschläge einer „Light“-Version des Bolognamodells den Markt der juristischen Ausbildungskonzepte werden bestücken wollen, zu denen etwa modularisierte Staatsexamensstudiengänge oder grundständige, aber „durchmodularisierte“ Masterstudiengänge, evtl. mit „Bachelorausgang“, gehören könnten.

Deren Einzelheiten bleiben abzuwarten. Obschon in der Diskussion oftmals bezeichnenderweise betont wird, die bisherigen Qualitätsmerkmale der Juristenausbildung müssten beibehalten werden, werden sich alle Änderungsmodelle daran messen lassen müssen, ob und inwieweit sie sowohl konzeptionell als auch unter den konkreten Handlungsbedingungen juristischer Fakultäten tatsächlich zu einer qualitativen Verbesserung der juristischen Ausbildung führen.

Melior est causa possidentis. Wer die Veränderung des Status quo anstrebt, trägt für Nutzen oder Notwendigkeit die Beweislast. 